Instituto Internacional de Investigación y Desarrollo Tecnológico Educativo INDTEC, C.A.

DOI: https://doi.org/10.29394/Scientific.issn.2542-2987.2019.4.12.5.104-126

OAI-PMH: http://www.indteca.com/ojs/index.php/Revista Scientific/oai

Artículo Original / Original Article

\title{
Las TIC's como Herramienta de Apoyo de la Estrategia Instruccional del Docente Universitario
}

\author{
Autores: Javier José Castro Capitillo \\ Universidad Nacional Experimental "Rafael María Baralt", UNERMB \\ jccapitillo@hotmail.com \\ Zulia, Venezuela \\ https://orcid.org/0000-0002-3946-705X \\ Jurismar Ivana Pérez Jiménez \\ Universidad Nacional Experimental "Rafael María Baralt", UNERMB \\ jurismarperez@gmail.com \\ Zulia, Venezuela \\ https://orcid.org/0000-0002-8866-6215 \\ Jurisbel Andreina Pérez Jiménez \\ Universidad Nacional Experimental "Rafael María Baralt", UNERMB \\ jurisbel357@hotmail.com \\ Zulia, Venezuela \\ https://orcid.org/0000-0003-0345-0042 \\ Blanca Leticia Caldera de Sánchez \\ Universidad Nacional Experimental "Rafael María Baralt", UNERMB \\ blancacaldera@hotmail.com \\ Zulia, Venezuela \\ https://orcid.org/0000-0002-4198-6748
}

\section{Resumen}

El objetivo del estudio fue analizar el uso de las tecnologías de comunicación como herramientas de apoyo de la estrategia instruccional aplicada por los docentes del Programa Administración de la Universidad Nacional Experimental "Rafael María Baralt" (UNERMB), sede Cabimas del Estado Zulia, como recurso de aprendizaje, enmarcado en una tipología analítica-descriptivo, diseño no experimental, transversal-De campo; utilizando una población de 46 docentes y 115 estudiantes, aplicando un censo poblacional en marzo del 2018. La técnica de recolección de los datos fue la observación mediante la encuesta, aplicando un cuestionario de 48 ítems, con cinco alternativas de respuestas, La validez y confiabilidad fue mediante el juicio de expertos, y el coeficiente de Alpha Cronbach, alcanzando un valor de 0.86 . El procesamiento de datos arrojó, para los docentes promedio de 3,15 y desviación de 0,62, indicando alto nivel de utilización de la estrategia instruccional, como recurso apoyada por las TIC's, para los estudiantes, el valor promedio fue de 2,80, con una desviación de 0,49 , reflejando un nivel moderado. Las diferencias en las respuestas entre docentes y estudiantes, con relación a las estrategias instruccionales y, la percepción del proceso metacognitivo del alumno, no han permitido consolidar, los conocimientos y elementos regulatorios que contribuyan al manejo efectivo de sus procesos cognitivos y, por consiguiente, a alcanzar un aprendizaje significativo, es por ello que se recomienda, evaluar las estrategias a fin contribuir con el logro de un aprendizaje significativo. educativa.

Palabras clave: tecnología de la comunicación; desarrollo tecnológico, innovación

Fecha de Recepción: 15-10-2018 Fecha de Aceptación: 19-02-2019 


\title{
ICTs as a Support Tool for the Instructional Strategy of the University Teacher
}

\begin{abstract}
The objective of the study was to analyze the use of communication technology as tools to support the instructional strategy applied by the teachers of the Administration Program of the National Experimental University "Rafael María Baralt" (UNERMB), headquarters of Cabimas, Zulia State, as a learning resource, framed in an analyticaldescriptive typology, non-experimental design, transverse-field; using a population of 46 teachers and 115 students, applying a population census in March 2018. The data collection technique was observation through the survey, applying a questionnaire of 48 items, with five alternative answers. The validity and reliability was through the judgment of experts, and the Alpha Cronbach coefficient, reaching a value of 0.86 . The data processing yielded, for average teachers 3.15 and deviation of 0.62 , indicating high level of use of the instructional strategy, as a resource supported by TIC's, for students, the average value was 2.80 , with a deviation of 0.49 , reflecting a moderate level. The differences in the responses between teachers and students in relation to instructional strategies and the perception of the student's metacognitive process have not allowed the consolidation of knowledge and regulatory elements that contribute to the effective management of their cognitive processes and, consequently, to achieving significant learning. This is why it is recommended to evaluate strategies in order to contribute to the achievement of significant learning.
\end{abstract}

Keywords: communication technology; technological development, educational innovation. 


\section{Introducción}

En el ámbito mundial, se viene gestando la revolución tecnológica y del conocimiento, lo cual ha generado profundos cambios en los procesos económicos, productivos, sociales, en los valores y creencias. Estas transformaciones, exigen a las instituciones educativas, innovaciones para acompañar estos procesos.

Ese marco general que contextualiza la educación mundial, en un entorno complejo en el cual las innovaciones tecnológicas impactan el funcionamiento de las organizaciones que conforman este importante sistema, no es diferentes para los países latinoamericanos, donde se ha generado una opinión generalizada que sostiene, según Tesdeco (2006): "los sistemas educativos no responden a esas demandas. Por ello, el tema educación ha venido ganando importancia, pasando a formar parte de grandes debates nacionales" (pág. 145); como: foros, conferencias, organizaciones comunitarias, ministerios y diversas instancias de la sociedad; asignándole el rol protagónico a su modernización y productividad; destacando la idea que la educación es responsabilidad del estado y sus ciudadanos, comprometido con el desarrollo sostenido del país y, su innovación debe comenzar a debatirse en cada entorno social de los centros educativos.

En ese sentido, la evolución histórica de la humanidad, ha demostrado que la educación se ha constituido en una herramienta para la transformación social y el mejoramiento sostenido de la calidad de vida y, en la misma esencia del hombre en su condición de ciudadano. Desde esa óptica, comprendiendo el importante papel que asumen los docentes, en su rol de agentes de la transformación; se les exige hoy día, prepararse para liderar las organizaciones educativas enmarcados en competencias que le den la fortaleza suficiente para educar individuos del futuro, requeridos por la sociedad del conocimiento.

Ese marco de retos y transformaciones, adquiere mayor complejidad en 
el nivel educativo universitario, al cual se le exige hoy la formación de profesionales con las competencias técnicas, humanas y éticas que requiere la sociedad, así como contribuir con el desarrollo científico-tecnológico a través de la investigación, marco dentro del cual las Tecnologías de Información y Comunicación (TIC), adquieren una mayor relevancia, pues la utilización de los sistemas computarizados y digitales a través del uso de software educativos, redes informáticas, digitales, entre otros, se ha convertido en herramientas de apoyo para la elevar la capacidad de autocrítica del estudiante, contribuyendo a elevar su procesos metacognitivos. En consonancia con lo anterior, para Romero (2012a):

La innovación tecnológica, en todas las áreas, de lo cual no escapa el sector educativo, posee una naturaleza cambiante y novedosa, pues contextualiza un entorno altamente dinámico, el cual se ha caracterizado por la implementación de estrategias de forma apresurada, asistemática y débilmente planificada (pág. 25).

Lo cual pudiera afectar la calidad de los procesos y la plena satisfacción de los grupos de interés internos y externos. Desde esta perspectiva, Romero (2012b), expresa que algunos: "fragmentos educativos conciben la tecnología con la innovación como algo que sólo las grandes y poderosas organizaciones pueden hacer y establecer, pues la relacionan directamente con la inversión costosa de plataforma y sofisticados equipos de punta" (pág. 31). Hoy día, la disponibilidad y ventajas que propicia la red, la capacidad de interacción y búsqueda continúa de información, plantea al docente y a la comunidad educativa en general, novedosas estrategias que propician la calidad educativa y el aprendizaje significativo de los estudiantes.

En ese orden de ideas, en el contexto educativo y, específicamente el universitario, la producción científica, ha contribuido con el desarrollo tecnológico, para propiciar la innovación y los elementos esenciales para orientar este importante proceso. En un marco de retos como el actual, donde 
la economía y del desarrollo social, adquieren fundamental relevancia, se ha venido planteando el desarrollo de los procesos de gestión tecnológica, de manera de crear e innovar el desarrollo de capacidades en el marco educacional, para generar una plataforma de apoyo para contribuir con la excelencia de las funciones de docencia, investigación y extensión.

Ante esa compleja realidad, los procesos de innovación se convierten en una respuesta a apremiante a las necesidades económicas-sociales y su impacto en las universidades, responsables hoy no solo de contribuir con la formación de profesionales con pertinencia y ética, sino también con la creación de una cultura basada en el desarrollo tecnológico como herramienta de transformación. Un ejemplo importante de esta tendencia, se observa en países como Estados Unidos, donde de acuerdo a Castellano (2012): "se han venido desarrollando plataformas tecnológicas de apoyo educativo, redes informáticas, mejoramiento de procesos, formación de competencias tecnológicas, necesaria para convertir las TIC's, en recursos de aprendizaje" (pág. 74); los cuales permitan propiciar los procesos metacognitivos de los estudiantes, pues se constituyen en pertinentes recursos instruccionales.

Se puede señalar, que ante el nuevo desafío de organizar la producción y aplicar TIC's como parte de la estrategia instruccional del docente, convierte a la innovación tecnológica en una de las capacidades con las que pueden contar las organizaciones académicas actuales. Desde esa reflexión, la innovación de la tecnología debe integrar elementos técnicos, económicos, educativos y humanos, con el fin de generar ventajas competitivas sostenibles a largo plazo, al contribuir con el mejoramiento de los procesos académicoadministrativos.

El ámbito universitario no escapa de esa realidad, pues la eficiencia del sector amerita el mejoramiento continuo de los procesos, asunto que debe convertirse en una prioridad de los procesos de gestión estratégica de del sector universitario y, debe estar dirigida hacia la búsqueda de la innovación de la estrategia instruccional, la apertura de procesos de transformación 
tecnológica y el desarrollo de las competencias de los docentes, de manera de convertirse en un elemento dinamizador del desarrollo y la transformación social, económica, política y cultural; sin embargo, las mayores dificultades se observan en las instituciones que cuentan con una disposición innovadora y carecen de un efectivo desarrollo de las TIC's, derivado entre otros factores, como la crisis presupuestaria actual, la desmotivación del personal docente y, en general la presencia de una cultura que no propicia el cambio como una necesidad de mejoramiento, convirtiendo la resistencia en una pauta de comportamiento.

Consustanciados con esa ideas, para Galeano (2013): "a pesar del potencial del sector universitario para contribuir a un incremento significativo de la calidad educativa, no se han realizado esfuerzos significativos que apunten al desarrollo e introducción de las TIC's" (pág. 114); como herramientas de aprendizaje, lo cual hace que pierdan cada vez más terreno y se constituyan en un sector emergente dentro de las estrategias de aprendizaje, en el cual las tecnologías de información y comunicación deben convertirse en recursos dinamizadores y potenciadores de las competencias de los estudiantes, de manera de alcanzar un aprendizaje significativo.

Asumir ese reto, característico de la posmodernidad, exigen a las instituciones educativas en general, asumir el reto de desarrollar las competencias tecnológicas del talento humano, invertir en sistemas de información y capacidades tecnológicas acordes con los nuevos desafíos; de ahí, la importancia que tiene el proceso de innovación y desarrollo tecnológico, pues esto implica el mejoramiento continuo de los procesos y, la posibilidad de elevar la calidad y excelencia educativa.

Desde esa visión de transformaciones y retos, la introducción de las TIC's en la praxis docente y la praxis educativa en general, permitirá aprovechar las oportunidad que ofrece el cambio, como mecanismos para reorientar la praxis y, generar un aprendizaje, de manera adaptarse a las 
nuevas situaciones y exigencias del mercado, desarrollo de nuevas competencias, redefinición de estrategias instruccionales por parte del docente, apertura de canales comunicacionales, entre otras condiciones que se conviertan en herramientas para elevar la calidad de las funciones universitarias.

De las situaciones enunciadas, se denota que uno de los desafíos más relevantes que vive la educación universitaria, dentro de la cuales las exigencias de calidad y pertinencia, se convierte en una prioridad de gestión. Al respecto, en los últimos años, adaptarse a los crecientes cambios tecnológicos, técnicos, científicos, sociales y culturales que surgen en la economía y la sociedad, se ha constituido en una prioridad de la gestión.

Ante estos cambios cada día más especializados, las TIC's, se presenta como la conexión más pertinente para dar respuesta a la necesidad de los participantes de formarse con altos niveles de calidad, para insertarse rápidamente en el mundo laboral, en un marco de transformación en la cual la información y comunicación se convierten en los recursos intangibles más valiosos de la de la sociedad del conocimiento, lo cual permite convertir las competencias profesionales e investigativas en herramientas de desarrollo de la sociedad del conocimiento.

Dentro de esa realidad, se encuentra el Programa Administración de la Universidad Nacional Experimental "Rafael María Baralt" (UNERMB), sede Cabimas, del estado Zulia, espacio académico donde se ha iniciado un proceso de modernización en la formación académica y andragógica, que contempla una renovación de su currículo, de su formación instruccional y forma de evaluación, para estimular el desarrollo científico con el propósito de aumentar y mejorar la calidad y excelencia académica.

Para ello, es necesaria una transformación en el área educacional de esta importante institución, donde el docente asume en su rol de mediador, investigador y promotor social, un papel relevante en la transformación y 
desarrollo de la universidad, pues su participación activa, su compromiso ineludible de superación y la competencia profesional, les permitirán asumir un reto de tal magnitud. Tal reto, será el de impulsar una educación de calidad, con apoyo de sistemas innovadores en todos los aspectos educativos y sus avances tecnológicos vanguardistas. Es necesario que los docentes sean los meros receptores en primera instancia y afines con estas tecnologías, situación que no está ocurriendo en este momento en la institución.

Por esa razón, se requieren docentes competentes, con las competencias técnicas, académicas, humanas y tecnológicas, necesarias para trasformar el modelo andragógico $y$, de forma directa la estrategia instruccional implementada dentro de su praxis, un profesional que cambie su comportamiento frente a la educación, su forma de pensar y de actuar; ayudándolo a usar nuevas estrategias tecnológicas basadas en TIC's, como recursos de aprendizaje, investigación y socialización.

De cara con lo expuesto y para ofrecer recomendaciones con respecto a la situación antes descrita, se hace necesario realizar el presente estudio, a fin de analizar las estrategias instruccionales aplicadas por los docentes universitarios en estudio, en el marco de la utilización de las TIC's, como recursos de aprendizaje. En esta línea argumental, en la búsqueda de evidencias que permitieran plantear la problemática, se aplicaron un conjunto de entrevistas diagnósticas no estructuradas, a una muestra de docentes, recabándose los siguientes signos y síntomas: Para los profesores, existe una importante resistencia al cambio; la cual se presenta en el uso de las TIC's; sin importar que en la actualidad las instituciones educativas, deben modernizarse y adaptarse a los cambios tecnológicos presentes para obtener mejores resultados y rendimientos en su utilización para con los estudiantes.

Dentro de esa dinámica de transformación, algunas organizaciones que se oponen de manera activa al cambio, manifiesta Chiavenato (2011), que: "oponerse al cambio, es tener un comportamiento negativo frente al cambio 
organizacional" (pág. 311); las organizaciones educativas o no, deben irse adaptando a los cambios tecnológicos que ocurren en su entorno; sin embargo, en algunos casos, cuanto más significativo es el cambio tecnológico, mayor resistencia por parte de los miembros.

Por otra parte, para Romero (2012c): "el docente manifiesta una actitud errónea hacia el uso de recursos instruccionales, basados en programas y medios tecnológicos, pues consideran que el enfoque tradicional aplicado, donde asume un rol dominante e impositivo" (pág. 38); convirtiéndose en un elemental transmisor de información. $Y$ en este orden de ideas, la introducción de las TIC's y renovación de la estrategia instruccional, termina produciendo hostilidad, escapándosele el monopolio de la transmisión de conocimientos.

Aunado a lo anterior, el mismo autor plantea que la resistencia hacia el uso de las TIC's, pudiera reflejar en grandes rasgos, analfabetismo tecnológico, y algún grado de temor por considerar que lo puede reemplazar en la transmisión de conocimientos, lo cual derivado de la resistencia al cambio, afianza el paradigma conductista, basado en la clase magistral, en el cual el profesor es el actor principal.

Se plantea en ese sentido, al profesor universitario posmoderno, una praxis académica basada en el uso de las TIC's, como recursos instruccionales, y sobrepasar de esa forma, las barreras de los sistemas tradicionales; dirigir la docencia hacia modelos de aprendizaje innovador, centrados en el aprendizaje del estudiante. Las TIC's, en ese sentido, se convierten en elementos de apoyo para potenciar procesos cognitivos. Lo anterior, exige la redefinición de la estrategia instruccional, a través de la incorporación de recursos que permitan propiciar la interactividad del estudiante, la incorporación de bases de datos y, de recursos que permitan propiciar la participación directa y autónoma del participante, a través de software instruccionales, video conferencias, entre otros recursos.

Se hace necesario en ese sentido, abrir un espacio de investigación y 
orientación, sobre el uso de las TIC's, para que estos docentes puedan brindar un mejor servicio y colocarse a tono con las exigencias del mundo de hoy. Es por ello, que se observa, una franca decadencia, marcada por la baja cobertura de profesores y al mismo tiempo, el desánimo para desarrollar sus competencias tecnológicas e innovar su estrategia instruccional.

Se observa, además que los docentes, han venido convirtiendo el acto educativo en un proceso monótono, donde prevalece la voz del profesor y su accionar el centro de todo el proceso; por tanto, las estrategias instruccionales son exposiciones magistrales, encontrándose ausente el empleo de equipos modernos tales como la Laptop, el Proyector de vídeo "video beam", las video conferencias, entre otros.

En razón a lo expuesto, se requiere la transformación del ejercicio andragógico del docente universitario, a través de la incorporación de las TIC's como recurso de aprendizaje, investigación y socialización; convirtiéndose en uno de los principales retos del proceso de formación del componente académico del profesor, por lo que se supone la actualización de los mismos, en estrategias instruccionales, que deberían permitir al profesorado educarse, en razón a los nuevos retos de la sociedad posmoderna, al concebir metodológicamente el uso de las TIC's como recursos de apoyo de las nuevas estrategias instruccionales.

En consonancia con lo descrito, el Ministerio del Poder Popular para la Educación Universitaria, Ciencia y Tecnología, hace grandiosos esfuerzos por mejorar la calidad educativa, mediante la capacitación docente; sin embargo, se refleja como una constante, la observación de situaciones difíciles en los profesores, al no asistir a las mismas e insistir en la utilización de metodologías monótonas, a las cuales los participantes no responden. Esto obedece a que los profesores no están formados bajo una concepción de avances en la ciencia, la técnica y el desarrollo de TIC's y este cambio vertiginoso, impacta en los diferentes procesos donde tienen lugar.

En el marco de esas ideas, el estudio estuvo orientado a analizar la 
estrategia instruccional aplicada por los docentes del Programa Administración de la UNERMB, sede Cabimas del Estado Zulia, en el marco del uso de la TIC's, como recursos de aprendizaje.

\section{Metodología}

Para lograr la rigurosidad científica, se requiere una clara visión del enfoque epistemológico que sustenta el estudio, de que las decisiones metodológicas tomadas durante el desarrollo de la investigación, mantengan coherencia y consistencia con el método seleccionado, el cual es de carácter deductivo, derivado de la posición positivista asumida en la investigación.

Al respecto, Cook y Richard (1995), citado por Hurtado (2010a): "el paradigma positivista, se caracteriza por la aplicación de un modelo hipotéticodeductivo, particularista, con énfasis en la objetividad" (pág. 18); y desde esa óptica en la condición deductiva del método, el cual asume la teoría como fundamento de la investigación.

En relación al tipo de investigación, el estudio se ubica dentro de la tipología analítica, definida por Hurtado (2010b): "como un proceso que profundiza la mera descripción, para lograr un nivel mayor, la comprensión profunda del evento, fenómeno o variable en estudio" (pág. 223); pues el análisis en un proceso reflexivo, lógico, cognitivo, orientado a la abstracción de pautas de relación interna de los componentes de evento, en un marco de análisis, que se convierte para la autora el criterio a través del cual se desarrolla.

Por otra parte, la investigación se apoyó en un diseño de investigación, no experimental, transeccional y de campo. En la investigación no experimental según Hernández, Fernández y Baptista (2010): "el investigador no tiene la intención de manipular el comportamiento de la variable, dimensiones e indicadores, sino únicamente hacer un registro de resultados" (pág. 215). En este tipo de diseño, no se realiza manipulación deliberada de la 
variable, pues su intención es solo registrar sistemáticamente su comportamiento natural.

Con relación a su condición de diseño transversal, la variable estudiada y su correspondiente criterio de análisis, se midieron en un momento determinado, razón por la cual, se recolectaron los datos en un solo momento, pues no se planteó de ninguna forma la intención de verificar su evolución.

Por su parte, la condición de campo del diseño, según Chávez (2009): alude a la utilización de "fuentes de información primaria, lo cual exige que el investigador se desplace al lugar específico donde se encuentran los informantes" (pág. 76).

La población de estudio estuvo conformada por los 46 docentes y 115 estudiantes del Programa Administración de la UNERMB. Al respecto, para la consideración de la población que conforma el universo, por su carácter finito; esta es considerada como mínima, pequeña y accesible, por lo tanto, se tomó un censo poblacional, definido por Arias (2012): el cual busca "recabar información acerca de la totalidad de una población" (pág. 33).

La técnica utilizada para la recolección de datos utilizada en la investigación fue la observación, la cual es definida por Méndez (2009): como "el proceso mediante el cual se observa la realidad a través de instrumentos o mecanismos específicos" (pág. 121); al respecto, por la condición deductiva del método positivista que sustenta la investigación, se partió de un esquema conceptual previo, del cual se abstrajeron las dimensiones e indicadores que se midieron a través del cuestionario, utilizado como instrumento de recolección de datos.

En cuanto al instrumento de recolección de datos se diseñó y aplicó un cuestionario, estructurado por 48 Ítems, con un escalamiento de cinco alternativas de respuestas: (5) Siempre; (4) Casi siempre; (3) Algunas veces; (2) Casi nunca; (1) Nunca, y, construido a partir de las dimensiones e indicadores que operacionalizaron la variable en estudio. Por otra parte, los 
datos fueron procesados a través del método de estadística descriptiva, utilizando para ello la técnica de medidas de tendencia central y variabilidad, a través del uso de promedio y la desviación estándar respectivamente, estadígrafos que fueron interpretados a través de baremos construidos por los investigadores.

\section{Resultado del estudio}

Tabla 1. Variable: Estrategias instruccionales (Resultado del estudio).

\begin{tabular}{|l|c|c|c|c|}
\hline \multirow{2}{*}{ Dimensiones } & \multicolumn{2}{c|}{ Docentes } & \multicolumn{2}{c|}{ Estudiantes } \\
\cline { 2 - 5 } & Promedio & Desviación & Promedio & Desviación \\
\hline $\begin{array}{l}\text { Estrategias } \\
\text { preinstruccionales }\end{array}$ & 3,27 & 0,54 & 2,62 & 0,47 \\
\hline $\begin{array}{l}\text { Estrategias } \\
\text { coinstruccionales }\end{array}$ & 3,21 & 0,68 & 2,78 & 0,45 \\
\hline $\begin{array}{l}\text { Estrategias } \\
\text { postinstruccionales }\end{array}$ & 2,98 & 0,64 & 3,01 & 0,54 \\
\hline Promedio general & $\mathbf{3 , 1 5}$ & $\mathbf{0 , 6 2}$ & $\mathbf{2 , 8 0}$ & $\mathbf{0 , 4 9}$ \\
\hline
\end{tabular}

Fuente: Los Autores (2018).

Con relación a la variable estrategia instruccional aplicada por los docentes, como se observa en la tabla 1, los resultados arrojados por el procesamiento de los datos, ubican las respuestas de los profesores en una categoría alta, al arrojar un promedio de 3.15 y una desviación de 0.62, indicando una categoría alta, con una muy baja dispersión de las respuestas, es decir, una alta confiabilidad, al manifestar que aplican para cada momento instruccional, a saber, antes, durante y terminada la sesión de aprendizaje, un conjunto de estrategias, técnicas y procedimientos que contribuyen a generar en los estudiantes, un aprendizaje significativo.

Por su parte, para los estudiantes, se evidencia un promedio en sus respuestas de 2.80, con una muy baja dispersión de 0.49 ; ubicándose en la categoría moderada; afirmando que con este nivel los docentes aplican las estrategias instruccionales que permitan orientar las actividades en el aula.

Los resultados contrastan con las aportaciones de Díaz (2010), al referir que: "las estrategias instruccionales incluyen de forma sistemática y ordenada, 
métodos, técnicas, procedimientos y recursos, los cuales deben ser planificados y ejecutados" (pág. 98); ajustados. En este marco de ideas, las actividades orientadas a la formación del personal, deben estar estrechamente relacionadas con su praxis, de manera de orientar el proceso de instrucción, sobre la base de los objetivos curriculares, la estrategia instruccional en particular, las necesidades particulares de cada estudiante, así como los recursos instruccionales con los cuales se cuenta, lo que da a la praxis instruccional, un orientación flexible y adaptable, como condición para elevar la calidad de los procesos.

Tabla 2. Variable: Uso de las TIC's

\begin{tabular}{|l|c|c|c|c|}
\hline \multirow{2}{*}{ Dimensiones } & \multicolumn{2}{|c|}{ Docentes } & \multicolumn{2}{c|}{ Estudiantes } \\
\cline { 2 - 5 } & Promedio & Desviación & Promedio & Desviación \\
\hline Recursos tecnológicos & 3,37 & 0,48 & 2,53 & 0,45 \\
\hline $\begin{array}{l}\text { Resistencia al uso de } \\
\text { las TIC's }\end{array}$ & 2,88 & 0,47 & 3,91 & 0,53 \\
\hline \multicolumn{1}{|c|}{ Promedio General } & $\mathbf{3 , 1 3}$ & $\mathbf{0 , 4 8}$ & $\mathbf{3 , 2 2}$ & $\mathbf{0 , 4 9}$ \\
\hline
\end{tabular}

Fuente: Los Autores (2018).

Corresponde presentar los datos arrojados por el procesamiento de los cuestionarios, en relación a la segunda variable, la cual representa de acuerdo a Hurtado (2010c): "el criterio de análisis, como soporte o fundamento de orientación del análisis" (pág. 325); relacionado en este caso, con el uso de las TIC's, como recursos de aprendizaje. Al respecto, como se observa en la tabla 2, el procesamiento de los datos para la variable, arrojó un promedio de 3.13, con una muy baja dispersión de 0.48; ubicándose en la categoría moderada; afirmando que con este nivel aplican los recursos tecnológicos y mantienen una resistencia al uso de las TIC's, dentro de su praxis instruccional $y$, como parte del desarrollo de la estrategia de aprendizaje que aplican a sus estudiantes.

Las respuestas de los docentes, se corresponden con la de los estudiantes al ubicarse en un promedio de 3.22, con una muy baja dispersión de 0.49 ; lo cual indica también una categoría moderada; al considerar que los 
profesores con este nivel aplican dentro de sus funciones los recursos tecnológicos para mediar el proceso de aprendizaje, así como mantienen cierta resistencia al uso de las TIC's, como lo expresa Zabala (2011):

Que las competencias en el desarrollo docente van mucho más allá de lo cognitivo, pues la educación contemporánea exige profesores altamente capacitados, que además de conocer o saber, tengan la capacidad de transmitir exitosamente sus conocimientos, ser ejemplo de conducta, carácter y personalidad, y además lograr motivar y conducir positivamente a los estudiantes hacia el cumplimiento de sus objetivos personales y profesionales (pág. 11).

En ese orden de ideas, el desarrollo de competencias tecnológicas y la incorporación de las TIC's como parte de la estrategia instruccional, contribuirá al fortalecimiento del proceso de aprendizaje colaborativo, la creatividad e innovación del participante y el despliegue de sus potencialidades.

En ese sentido, para los profesores y estudiantes dentro de la institución se utilizan de forma moderadas las herramientas computacionales como elementos de apoyo para ejecutar su estrategia instruccional. Consideran también que con ese nivel se aprovechan las oportunidades que presentan los adelantos tecnológicos para trasmitir mayor cantidad de información en periodos de tiempo donde la rapidez se convierte en una prioridad y, se utiliza la tecnología de información y comunicación como una herramienta para trasmitir información a los estudiantes ubicados en lugares diferentes a la universidad.

En ese orden de ideas, Espinoza (2014a), la utilización de recursos instruccionales, apoyados por la utilización de las TIC's: "apertura la posibilidad de integrar herramientas como la inmaterialidad, derivada del carácter intangible de la información, además de una posibilidad para construir mensajes" (pág. 121); derivado de su capacidad para procesar información grandes niveles y cantidades de información, lo cual complejiza el proceso 
educativo y el aprendizaje, facilitar el acceso a la misma información con códigos lingüísticos diferentes, transmitirla a destinos lejanos, con costos menores y en tiempo real.

En ese orden de ideas, para docentes y estudiantes, como moderada frecuencia se utilizan aplicaciones tecnológicas que facilitan experiencias de aprendizaje auto-administradas, así como herramientas tecnológicas que permiten establecer el ritmo del proceso de aprendizaje, así como la profundización de la información manejada en las sesiones de aprendizaje, lo cual, de acuerdo al autor citado, refiere condición de interactividad.

Al respecto, para Romero (2012d): "la condición de interactividad que ofrece la innovación tecnológica, contribuye no sólo elaborar mensajes, sino que, además, poder decidir la secuencia, establecer el ritmo, cantidad y nivel de profundidad" (pág. 87); cuando el usuario puede elegir el tipo de código con el que quiere establecer relaciones con la información se desarrolla su capacidad de autocontrol, aspecto básico a la hora de operacionalizar una estrategia de aprendizaje.

Por otra parte, para los profesores y estudiantes, en la institución, se utiliza de forma moderada dentro de la estrategia instrucción, las ventajas ofrecidas por los procesos de digitalización para sistematizar las experiencias de aprendizaje. Consideran también que demuestran una moderada capacidad para digitalizar imágenes que contribuyen con los procesos de aprendizaje y, cuentan de forma moderada con los recursos tecnológicos que le permiten aprovechar las ventajas de la digitalización en el proceso de aprendizaje.

Para Espinoza (2014b): "las representaciones digitales de imágenes, sonidos o vídeos, permite al usuario utilizar de forma integral sus sistemas de recepción de información" (pág. 45); visuales, auditivos y kinestésicos, lo cual contribuye con la transmisión, almacenamiento y recepción de muchos tipos de información. Esto convierte a los sistemas digitales y el uso de las TIC's, 
en efectivas herramientas de aprendizaje.

En otro orden de ideas, los docentes y estudiantes, manifestaron utilizar de forma moderadas tecnologías de información y comunicación (TIC) que favorecen la integración de entidades de conocimiento, pues según su apreciación se constituyen en herramientas de innovación de los procesos de aprendizaje. Expresaron, además, que utilizan de forma moderada el Internet como herramienta estándar de comunicación fundamental para acceder al conocimiento. En este marco, Romero (2012e): expone que "la interconexión permite formar una nueva red de comunicación que conduce al máximo aprovechamiento de las tecnologías como herramientas de aprendizaje" (pág. 92); y mecanismos de desarrollo de los procesos metacognitivos de los estudiantes.

\section{Conclusiones}

La sociedad posmoderna del siglo XXI, donde la información y comunicación se han convertido en la más importante propiedad intelectual y mecanismo de crecimiento y transformación, plantean una dinámica de cambios constantes, ha tomado mucha importancia las innovaciones tecnológicas como herramientas de trabajo, comunicación y aprendizaje.

Es por ello, que se hace importante abordar desde la perspectiva científica, temas importantes como el uso de la TIC's, en la educación, para brindar a la comunidad universitaria una plataforma educativa, instruccional, administrativa e investigativa, que contribuya a elevar la calidad y excelencia académica.

Ante esta realidad, la integración innovadora entre las tecnologías y la educación, se convierten en un factor de desarrollo y mejoramiento de los procesos instruccionales. El reto de hoy, no es solo la modernización de la plataforma tecnológica, sino también la preparación de la comunidad educativa en general, de manera de dirigir su praxis hacia un cambio que valide y 
satisfaga las necesidades de la sociedad, se convierte en una herramienta de apoyo y consolide una cultura comprometida con la calidad y excelencia.

En el marco de esas ideas, la contrastación de las respuestas de las fuentes de información, revelan en la unidad de estudio, una moderada aplicación de los tipo de estrategias instruccionales, en cada una de las fases del proceso (antes, durante y después de la sesión de aprendizaje), así como una moderada aplicación de los recursos tecnológicos apoyados por las TIC's, con un nivel también moderada de resistencia, generado no solo para el nivel de desarrollo de las competencias tecnológicas de los profesores y estudiantes, sino también por las deficiencias presupuestarias generadoras de condiciones de desactualización, restricciones, entre otros.

Se refleja en ese sentido, que los estudiantes, actores principales del proceso y clientes fundamentales del proceso instruccional, perciben un moderado uso de las TIC's, como herramientas o recursos instruccionales, en cada una de las fases de la estrategia, lo cual conduce a la utilización de mecanismos que no permiten la utilización de los sistemas de recepción del estudiante, el desarrollo de su capacidad creativa y el manejo de los niveles motivacionales, esenciales para lograr un aprendizaje significativo.

\section{Referencias}

Arias, F. (2012). EI Proyecto de Investigación. Introducción a la metodología científica. 6ta edición, ISBN: 980-07-8529-9. Caracas, Venezuela: Editorial Episteme, C.A. Recuperado de:

http://www.colegioiberoamericano.edu.ve/pdf/FidiasAriasproyecto Invest 6taEdic.pdf

Castellano, R. (2012). La nueva era de la educación. México: Editorial Limusa.

Chávez, N. (2009). Introducción a la investigación educativa. ISBN: 980295-068-8. Maracaibo, Venezuela: Gráfica González, 325 págs. 
Chiavenato, I. (2011). Gestión del talento humano. Tercera Edición. México, D.F.: Editorial McGraw-Hill Interamericana S.A.

Díaz, F. (2010). Estrategias docentes para su aprendizaje significativo. ISBN: 978-970-10-7340-7. México, D.F.: Mcgraw-Hill/Interamericana Editores, S.A. DE C.V. Recuperado de:

http://www.facso.unsj.edu.ar/catedras/cienciaseconomicas/administracion-de-personal-1/documentos/chiavena.pdf

Espinoza, R. (2014a,b). Principios tecnológicos en la educación. Colombia:

Editorial Norma.

Galeano, D. (2013). La innovación en la educación. México, D.F.: Editorial McGraw-Hill Interamericana S.A.

Hernández, R., Fernández, C., \& Baptista, P. (2010). Metodología de la investigación. Quinta Edición, ISBN: 978-607-15-0291-9. México, D.F.: Editorial McGraw-Hill Interamericana S.A.

Hurtado, J. (2010a,b,c). El proyecto de investigación. Comprensión holística de la metodología y la investigación. 6ta. Edición. Caracas, Venezuela: Ediciones Quirón-Sypal.

Méndez, C. (2009). Metodología de la investigación. México D.F.: Editorial McGraw-Hill Interamericana S.A.

Romero, R. (2012a,b,c,d,e). Estrategias en el aula para el desarrollo del aprendizaje en estudiantes universitarios. Teoría y Práctica. México, D.F.: Editorial Trillas.

Tesdeco, J. (2006). La educación en América Latina y El Caribe. México, D.F.: Editorial Trillas.

Zabala, M. (2011). Competencias docentes del profesorado universitario. Calidad y desarrollo Profesional. 2da Edición. Madrid, España: Narcea Ediciones, 228 págs. 


\section{Javier José Castro Capitillo}

e-mail: jccapitillo@hotmail.com

Nacido en Cabimas, Estado Zulia, Venezuela. Licenciado

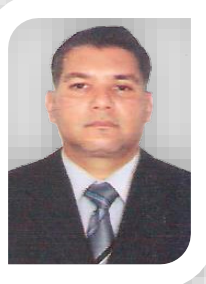

en Administración, Mención Gerencia Industrial en el año 1999 en la Universidad Nacional Experimental "Rafael María Baralt" (UNERMB); Magister Scientiarum en Gerencia de Recursos Humanos (UNERM, 2010); Especialización de Docencia Universitaria en el año 2012; y Doctor en Ciencias Gerenciales en el año 2016 en la Universidad "Dr. Rafael Belloso Chacín"; Formación Docente para Egresados - Componente Docente (UNERMB, 2017). Actualmente me desempeño en la UNERMB, como Docente de las Materias: Gerencia de Mercado, Taller de Acción Profesional I y II, Matemáticas II, Estrategias Gerencial. Finanzas de la Empresa, Fundamentos Matemáticos y Aritmética I. 


\section{Jurismar Ivana Pérez Jiménez \\ e-mail: jurismarperez@gmail.com}

Nacida en el Municipio Cabimas, Estado Zulia,

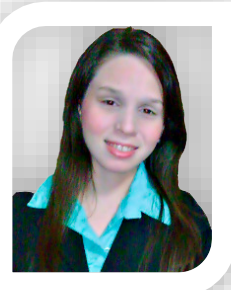

Venezuela. Licenciada en Educación Mención

Informática, de la Universidad del Zulia en el año 2013;

Magister Scientiarum en Gerencia de Recursos

Humanos, egresada de la Universidad Nacional

Experimental "Rafael María Baralt" en el año 2016.

Docente en Educación Media Técnica de la E.T.C.R. "Hermágoras Chávez", además, Coordinadora, tutora y asesora de varios trabajos de Pasantías de este nivel. 


\section{Jurisbel Andreina Pérez Jiménez}

e-mail: jurisbel357@hotmail.com

Nacida en el Municipio Cabimas, Estado Zulia,

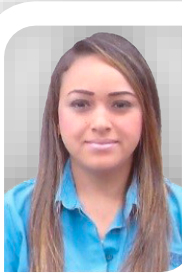

Venezuela. Licenciada en Educación Mención

Informática, de la Universidad del Zulia en el año 2010;

Magíster Scientiarum en Informática Educativa en la

Universidad Privada Dr. Rafael Belloso Chacín (URBE,

2013); Diplomados en: Experto en Medios y Periodismo

Digital en el año 2017; Experto en Comercio Electrónico en el año 2016; y Experto en Procesos Elearning en el año 2013 en la Fundación para la actualización tecnológica de Latinoamérica (FATLA); Experiencia laboral: Gerente de Sistemas en Dimaica Maturín, C.A. en el año 2016; Docente Contratada en la UNERMB en el año 2014; Coordinadora de Informática en la U.E.P. Colegio "Virgen del Rosario" en el año 2014. Actualmente trabajo para la Alcaldía de Cabimas como programadora. 


\section{Blanca Leticia Caldera de Sánchez}

e-mail: blancacaldera@hotmail.com

Nacida en el Municipio Cabimas, Estado Zulia,

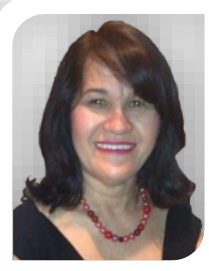

Venezuela. Médico especialista en Pediatría y

Puericultura, egresada de la Universidad del Zulia en el año 1980; Docente universitario de la asignatura Clínica Pediátrica, categoría agregado, con dedicación medio tiempo, núcleo Cabimas. Actualmente soy Coordinadora de la Clínica Pediátrica de la Unidad Docente Cabimas, en el Hospital General de Cabimas "Dr. Adolfo D'Empaire". 